hep-th/9605199

RU-96-41

\title{
Probing F-theory With Branes
}

\author{
Tom Banks \\ Michael R. Douglas \\ Nathan Seiberg \\ Department of Physics and Astronomy \\ Rutgers University \\ Piscataway, NJ 08855-0849 \\ banks, mrd, seiberg@physics.rutgers.edu
}

Last week, A. Sen found an explicit type I string compactification dual to the eightdimensional F-theory construction with $S O(8)^{4}$ nonabelian gauge symmetry. He found that the perturbations around the enhanced symmetry point were described by the mathematics of the solution of $\mathcal{N}=2, d=4 S U(2)$ gauge theory with four flavors, and argued more generally that global symmetry enhancement in $\mathcal{N}=2, d=4$ gauge theories corresponded to gauge symmetry enhancement in $F$-theory.

We show that these $\mathcal{N}=2, d=4$ gauge theories have a physical interpretation in the theory. They are the world-volume theories of 3-branes parallel to the 7 -branes. They can be used to probe the structure of the exact quantum $F$-theory solutions. On the Higgs branch of the moduli space, the objects are equivalent to finite size instantons in the 7-brane gauge theory.

May 1996 


\section{Introduction}

$F$-theory is a powerful approach to superstring compactification, which has been the focus of much recent work [1-7]. Although there are strong hints that a twelve-dimensional definition of the theory exists, at present its clearest definition is as the class of type IIb string compactifications containing 7-branes, which generically have non-constant dilaton and axion fields. These fields can be represented as the modulus of an elliptic curve fibered over the space-time, and thus the basic structure of the compactification is given by a complex manifold admitting an elliptic fibration. Singularities of the fiber will correspond to positions of 7 -branes, and encircling one of these induces an $S L(2, \mathbb{Z})$ monodromy on the IIb fields.

The prototypical example is the representation of the surface $K 3$ as an elliptic curve fibered over $\mathbb{P}^{1}$. The moduli space of such fibrations is 18 complex dimensional and isomorphic to the Narain moduli space of heterotic string compactifications of $T^{2}$. This and other evidence suggests that the theories are dual. By the adiabatic argument, it follows that compactifications on $n+1$-dimensional complex manifolds are dual to heterotic string compactifications on $n$-dimensional manifolds with a specific choice of gauge background. This unification of gauge and metric parameters is a significant advantage of the approach.

In a beautiful recent work, Sen has found an explicit heterotic dual to an $F$-theory compactification [8]. The starting point is the observation that there is a special elliptically fibered $K 3$ for which the $S L(2, \mathbb{Z})$ monodromies are almost trivial, and the dilaton and axion fields constant - the orbifold $T^{4} / \mathbb{Z}_{2}$. Regarding it as a $T^{2}$ fibration over $T^{2} / \mathbb{Z}_{2}$, the $T^{2}$ complex structure is obviously constant. Thus there should be no barrier to regarding this as a conventional type $\mathbb{I b}$ compactification.

In fact it is an orientifold compactification. The $S L(2, \mathbb{Z})$ monodromy around a $\mathbb{Z}_{2}$ fixed point of the orbifold as a fibration is $\mathbf{- 1}$. This has the same action on the massless fields as $(-1)^{F_{L}} \Omega$, and thus we can regard the compactification as an orientifold by the $\mathbb{Z}_{2}$ transformation $(-1)^{F_{L}} \Omega R$, where $R$ is $z \rightarrow-z$ on the base $T^{2}$.

This is known as a type $\mathrm{I}^{\prime}$ compactification [9], in other words one produced from the type I string by $T$-duality of both coordinates of the base. The transformation $(-1)^{F_{L}} \Omega R$ is mapped to $\Omega$, so the compactification is mapped to a conventional type I theory with enhanced gauge symmetry $S O(8)^{4}, S$-dual to the heterotic string. It has four 7-branes inserted at each of four orientifold fixed points, and nonabelian gauge symmetry $S O(8)^{4}$.

Given the duality between $F$-theory at the orbifold point and the calculable type I theory or type $\mathrm{I}^{\prime}$ orientifold, it is now possible to vary parameters in the type $\mathrm{I}^{\prime}$ description and learn about $F$-theory moduli space. Sen's next observation is that quantum effects 
are always important in this problem, and that the classical dilaton-axion solution of the form described in [10] is modified.

The mathematics of $F$-theory strongly suggests, and Sen argues convincingly, that the modification is the same as the passage from the one-loop result to the finite coupling solution of an $\mathcal{N}=2, d=4 S U(2)$ gauge theory with four fundamental hypermultiplets. The parameters and moduli of this gauge theory are the masses $m_{i}$ of the hypermultiplets and the vev $z=\left\langle\operatorname{tr} \phi^{2}\right\rangle$ of the adjoint scalar, which are identified with positions of 7branes and a point in the base (locally $\mathbb{R}^{2} / \mathbb{Z}_{2}$ ). The gauge coupling $\tau$ is identified with the dilaton-axion, and the gauge theory solution provides its dependence on $z$ and the parameters.

In this note we point out that all this has a simple physical explanation. One way to study the properties of a background in string theory is to study the effective Lagrangian of an object (or 'probe') moving in that background. The background fields will turn into couplings in this Lagrangian, but in general these are not directly observable - only physical observables including possible quantum corrections are meaningful.

If the background is defined by a configuration of D-branes, and the probe is a D-brane, instead of solving for the bulk fields produced by the background configuration, there is another way to study these effects. The combined system of D-branes will have various fields corresponding to open strings stretched between pairs of D-branes. Strings ending on the probe will correspond to fields on its world-volume, and closed string backgrounds will produce couplings in its Lagrangian. Solving for the dynamics of the probe will then reproduce the effect of the background field configuration. This has been demonstrated in the example of the D5-brane as instanton [11], by using a 1-brane as probe [12]. Further examples will appear in [13], along with a study of the regime of validity of this approach.

In the $F$-theory context, the 3 -brane is the natural probe, as 7-branes parallel to the 3-brane will break only half its supersymmetry, leaving an $\mathcal{N}=2, d=4$ worldvolume Lagrangian. Furthermore, the 3-brane is the only IIb brane which is a singlet under $S L(2, \mathbb{Z})$ (which becomes the $S L(2, \mathbb{Z})$ duality symmetry of its world-volume theory), making it canonically defined in any $F$-theory situation. Finally, Sen's results tell us that an $\mathcal{N}=2, d=4$ gauge theory will naturally encode much of the physics. The spacetime variation of the dilaton-axion fields in F-theory is mapped onto the variation of the low energy gauge coupling function on moduli space. We will see that this has the following simple physical interpretation: As a 3-brane is moved around in spacetime, the coupling of the gauge theory on its world volume is determined by the background fields. The adjoint VEV which parametrizes the moduli space of the world volume gauge theory is simply the 3-brane position, relative to the orientifold point of the type $\mathrm{I}^{\prime}$ theory. Furthermore, 
it is now easy to understand the appearance of $S U(2)$ gauge theory. The 5-brane of type I theory comes equipped with $S U(2)$ Chan-Paton factors 11,14. By T-duality these are inherited by the type $\mathrm{I}^{\prime} 3$-brane. The five brane $\mathrm{T}$ dual to our 3 -brane probe is wrapped around the two torus. A Wilson line in its world volume $S U(2)$ gauge theory breaks the gauge group down to $U(1)$. In the $\mathrm{T}$ dual theory, the 3 -brane lives in the noncompact dimensions and can move around on the torus. $S U(2)$ symmetry is restored only when it approaches one of the orientifold points. For other configurations, the massive charged gauge bosons are realized as open strings connecting the 3 brane to the orientifold point.

A final aspect of Sen's result which is clarified by our analysis is the fact that the gauge symmetries of string theory showed up as global symmetries of the $S U(2)$ gauge theory which describes the curve. Gauge symmetries always appear as global symmetries in the effective lagrangian of an extended object. This is necessary for consistent coupling of that object to background gauge fields.

\section{Type $\mathbf{I}^{\prime}$ orientifold and $F$-theory near an orbifold point}

We start with the equivalent type I theory on $T^{2}$ with gauge symmetry broken by Wilson lines with holonomy $\exp 2 \pi i \sum_{i} \alpha_{i, \mu} R_{i}$, with $1 \leq i \leq 16, \mu \in\{8,9\}$ and $R_{i}$ generators of $U(1)^{16} \subset S O(32)$. Explicit Wilson lines breaking to $S O(8)^{4}$ are $\alpha_{4 j+i, 8}=j / 2$ and $\alpha_{8 k+i, 9}=k / 2$. The complete gauge symmetry includes $U(1)^{4}$ as well from the metric and $C^{(2)}$ fields on $T^{2}$.

Introduce a 5 -brane transverse to the $T^{2}$, the $T$-dual of the 3 -brane of the introduction. Its world-volume theory is $\mathcal{N}=1, d=6 S p(1)$ gauge theory, which on $T^{2}$ has Wilson line moduli $\mathcal{A}_{\mu}^{a}$ with eigenvalues $\theta_{\mu}$. It also contains $32 S p(1)$ doublet 'half-hypermultiplets' [11]. These will be given mass by the Wilson lines. The matter can be rewritten as 16 doublet hypermultiplets with masses $\left(\alpha_{i, 8}+i \alpha_{i, 9}\right)-\left(\theta_{8}+i \theta_{9}\right)$. The maximal massless matter content is 4 hypermultiplets attained by taking $\theta_{8}=\theta_{9}=0$.

The $F$-theory limit is the limit of small $T^{2}$, in which momentum modes in the $T^{2}$ decouple. After $T$-duality of both of these dimensions the Wilson line parameters become positions on the dual $T^{2} / \mathbb{Z}_{2}$. Call the complex positions of the 7 -branes $m_{i}=\left(R_{8}^{\prime} \alpha_{i, 8}+\right.$ $\left.i R_{9}^{\prime} \alpha_{i, 9}\right)$ and the complex position of the 3-brane $A=A^{a} \sigma_{a}=\left(R_{8}^{\prime} \mathcal{A}_{8}+i R_{9}^{\prime} \mathcal{A}_{9}\right)$. This can be diagonalized to

$$
A=\left(\begin{array}{cc}
w & 0 \\
0 & -w
\end{array}\right),
$$

the expectation value of an adjoint Higgs breaking $S p(1)$ to $U(1)$. The gauge-invariant parameter is $z=\frac{1}{2} \operatorname{tr} A^{2}=w^{2}$. 
As in [14] it is convenient to work on the covering space $T^{2}$, introducing a $\mathbb{Z}_{2}$ image for each brane. From now on we focus on the neighborhood of a single $\mathbb{Z}_{2}$ fixed point, $w=0$, and ignore the 7 -branes far from the fixed point. The images are then located at $-m_{i}$ and $-w$. The pair of 3-branes at $(w,-w)$ are the same pair introduced in type I theory to get $S p(1)$ and these images lead to the structure (2.1), while the two 7-brane images each give open strings in half-hypermultiplets. At tree level, the half-hypermultiplets have the $(\mathcal{N}=1)$ superpotential

$$
W=\sum_{i} m_{i} h_{i} \tilde{h}_{i}+\operatorname{tr} h_{i} A \tilde{h}_{i}
$$

and obtain masses $m_{i}+w$ and $m_{i}-w$.

In the gauge theory context, the next step is to compute the perturbative renormalization in the $U(1)$ theory with charged matter. This produces the effective gauge coupling

$$
\tau(z)=\tau_{0}+\frac{1}{2 \pi i}\left(\sum_{i=1}^{4} \log \left(z-m_{i}^{2}\right)-4 \log z\right) .
$$

The cutoff would a priori be string scale but in fact cancels in this finite theory. This is clear from the alternate interpretation of the result as the dilaton-axion produced by the 7-brane sources, probed by the 3-brane.

As Sen points out, this result is physically unacceptable as it does not satisfy $\operatorname{Im} \tau \geq 0$ everywhere in the complex plane. At strong coupling, D-instanton corrections, which behave as $e^{-\tau}$, will become large. These are equivalent to the 3-brane gauge theory instantons and the exact quantum result for $\tau$ is that given in [15].

This leaves the question of why the masses of open strings stretching from one 7-brane to another are given by the simple formulae $m_{i} \pm m_{j}$. Properly speaking, this is a question of 7-brane quantum dynamics, but we can infer the answer from our results by a simple trick. The strings stretching from the 3-brane to a 7-brane are just the matter multiplets whose masses are given by $m_{i} \pm w$. Now take the 3-brane to the point $w=m_{j}$. The masses of these strings will then be $m_{i} \pm m_{j}$. But a 37 and a 77 string are both BPS saturated, and both couple to the bulk fields in exactly the same way. The central charge and therefore the mass must be the same for both. We have thus reproduced all of Sen's results from the physics of 3-branes.

\section{Discussion}

Sen has made a link between $F$-theory and the quantum dynamics of 7 -branes. We used a 3-brane to see these quantum effects, and argued that the quantities studied by Sen, 
the dilaton-axion and BPS masses, had observable counterparts on the 3-brane. However the effects would be present without the 3-brane. This leads to the question: can we understand the quantum dynamics of the 7-branes more directly?

The same object, the D-instanton, is responsible for the non-perturbative corrections in both 3-brane and 7-brane theories. In the 3-brane context it is equivalent to the gauge instanton, whose effects are known. The low energy field theory of the 7-brane is not renormalizable, but perhaps nonrenormalization theorems would allow us to perform an exact field theoretic computation of certain quantities.

The 3-brane coupling $\tau$ can go to zero at certain points, and naively one might have interpreted this as the tension going to zero and the 3-brane becoming light. However, one should do an $S L(2, \mathbb{Z})$ transformation to weak coupling to see the real situation.

The approach is clearly very general. One can repeat the analysis for general D-brane configurations at general orbifold singularities to get more general matter. Qualitative properties of the 3-brane gauge dynamics will translate directly into properties of the geometry. For example, with more than four 7-branes at the fixed point, the theory will be IR free, and the orientifold point will not split.

When several 7-branes coincide the eight dimensional space time theory has enhanced non-Abelian gauge symmetry, and thus has Yang-Mills instanton solutions. These will be BPS saturated 3-branes which are expected to be equivalent to the Dirichlet 3-brane on an appropriate branch of its moduli space [16]. Indeed, the 3-brane theory has a corresponding enhanced global symmetry at these points, and a Higgs branch. Moving along the Higgs branch, the instanton size grows. This interpretation can be justified in several different ways. For example, in the type I theory, a space time instanton is on the Higgs branch of the D5-brane. As this brane wraps the two torus, it leads to our 3-brane whose Higgs branch is this instanton. $\mathcal{N}=2$ supersymmetry implies that the metric on this branch does not receive quantum corrections [15, 17].

In this context, it will be interesting to search for gauge theories with $E$-series global symmetry, to explain the $E_{8}$ gauge symmetry of the heterotic string. This symmetry might arise non-perturbatively at special points in moduli space. We have to vary the base to get these symmetries, and a logical place to search for it is at other orbifold points $\mathbb{R}^{2} / \mathbb{Z}_{n}$.

One could also introduce more than one 3-brane, to get more general gauge groups. We could no longer regard a single 3-brane in this system as an isolated probe, but clearly this is a very simple way to produce interesting $d=4$ gauge theories. There are a host of further generalizations of these ideas. For example, we can consider type I on $K 3$ and wrap a five brane on a two cycle. The resulting 3 -brane in six dimensions has $\mathcal{N}=1$ supersymmetry. There can be an interesting interplay between the dynamics of this theory and the background it propagates in. 


\section{Acknowledgements}

This work was supported in part by DOE grant DE-FG02-96ER40559 and NSF grant PHY-9157016. We thank A. Sen, S. Shenker and E. Witten for discussions. 


\section{References}

[1] C. Vafa, "Evidence for F-theory," hep-th/9602022.

[2] D. Morrison and C. Vafa, hep-th/9602114.

[3] D. Morrison and C. Vafa, hep-th/9603161.

[4] N. Seiberg and E. Witten, hep-th/9603003.

[5] E. Witten, hep-th/9603150; hep-th/9604030.

[6] S. Ferrara, R. Minasian and A. Sagnotti, hep-th/9604097.

[7] P. Aspinwall and M. Gross, hep-th/9605131.

[8] A. Sen, "F-theory and Orientifolds," hep-th/9605150.

[9] S. Chaudhuri, C. Johnson, and J. Polchinski, "Notes on D-Branes," hep-th/9602052.

[10] B. Greene, A. Shapere, C. Vafa and S.-T. Yau, Nucl. Phys. B337 (1990) 1;

G. Gibbons, M.B. Green and M.J. Perry, hep-th/9511080.

[11] E. Witten, "Small Instantons in String Theory," hep-th/9511030.

[12] M. R. Douglas, "Gauge Fields and D-branes," hep-th/9604198.

[13] M. R. Douglas, D. Kabat, P. Pouliot and S. H. Shenker, to appear.

[14] E. G. Gimon and J. Polchinski, "Consistency Conditions for Orientifolds and Dmanifolds," hep-th/9601038.

[15] N. Seiberg and E. Witten, Nucl. Phys. B431 (1994) 484, hep-th/9408099.

[16] M. R. Douglas, "Branes within Branes," hep-th/9512077.

[17] P.C. Argyres, M.R. Plesser and N. Seiberg, hep-th/9603042. 